\title{
Genetic and Pathogenetic Aspects of Noonan Syndrome and Related Disorders
}

\author{
Martin Zenker \\ Institute of Human Genetics, University Hospital Erlangen, University of Erlangen-Nuremberg, Erlangen, and \\ Institute of Human Genetics, University Hospital Magdeburg, Otto-von-Guericke University, Magdeburg, Germany
}

\section{Key Words}

Noonan syndrome $\cdot$ Short stature $\cdot$ Tumor predisposition • RAS-MAPK signaling $\cdot$ Neurofibromatosis

\begin{abstract}
Noonan syndrome (NS) and the clinically overlapping disorders cardio-facio-cutaneous syndrome, LEOPARD syndrome, Costello syndrome and Neurofibromatosis-Noonan syndrome share the clinical features of short stature, the same spectrum of congenital heart defects, and a similar pattern of craniofacial anomalies. It is now known that all these disorders are caused by mutations in components of the RASMAPK signaling pathway. This pathway was previously known for its involvement in tumorigenesis. This article reviews the current knowledge on underlying genetic alterations and possible pathogenetic mechanisms responsible for NS and related disorders. It discusses the relationship between a group of developmental disorders and oncogenes. Potential future treatment prospects are based on the possibility of inhibiting RAS-MAPK signaling by pharmaceuticals.

Copyright @ 2009 S. Karger AG, Basel
\end{abstract}

\section{Noonan Syndrome and Related Disorders - The Neuro-Cardio-Facial-Cutaneous Syndrome Spectrum}

Noonan syndrome (NS) was named after the pediatric cardiologist Jacqueline Noonan, who first proposed this condition as a separate entity in 1968 [1]. The clinical hallmarks of NS are: (1) congenital heart defects, (2) short stature, and (3) typical facial anomalies. Cardiac anomalies include valvular pulmonic stenosis as the most common heart defect, hypertrophic cardiomyopathy and septal defects $[2,3]$. Short stature is of postnatal onset, proportionate, and usually of mild-to-moderate degree. The typical craniofacial anomalies are illustrated in figure 1a. In addition, patients with NS often show chest deformities and ametropia. Lymphatic anomalies may present prenatally as nuchal edema, pleural effusions or hydrops fetalis, and postnatally with hand or foot edema. More than half of the individuals affected by NS show some delay in developmental milestones (motor and/or speech delay), and roughly one third exhibit learning difficulties and require special educational support. However, mental retardation (IQ $<70)$ is uncommon in patients with NS. Leukemia, in particular juvenile myelomonocytic leukemia (JMML), has been

\section{KARGER \\ Fax +4161306 1234 \\ E-Mail karger@karger.ch}

www.karger.com (c) 2009 S. Karger AG, Basel

0301-0163/09/0728-0057\$26.00/0

Accessible online at:

www.karger.com/hre
Martin Zenker

Institute of Human Genetics, University Hospital Magdeburg, Leipziger Str. 44 DE-39120 Magdeburg (Germany)

Tel. +49391671 5062, Fax +493916715066

E-Mail martin.zenker@med.ovgu.de 
Fig. 1. Facial appearance of a male patient with NS due to a SOS1 mutation (a), a male patient with CFCS due to a BRAF mutation (b), and a female affected by CS carrying a HRAS mutation (c). Note the similarities with hypertelorism, down-slanting palpebral fissures, ptosis, broad forehead and low-set ears. Curly hair and sparse eyebrows are prominent in the CFCS patient, while coarse facial appearance stands out in the CS patient. Image $\mathbf{c}$ is courtesy of Sabine Uhrig, Graz.
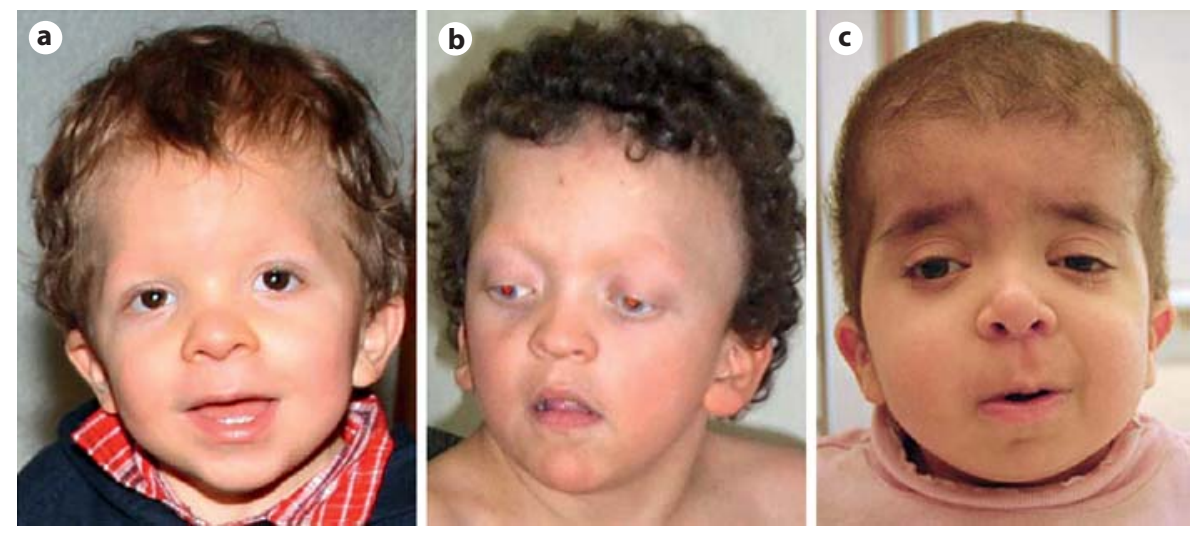

observed occasionally in patients with NS, but the overall risk of JMML in NS is assumed to be less than $1 \%$ [4].

The term cardio-facio-cutaneous syndrome (CFCS) was coined by Reynolds et al. [5] in 1986. They described a condition that obviously shared the major clinical features of NS (fig. 1b), but differed from NS mainly in the high frequency of ectodermal anomalies (hyperkeratotic skin changes; thin, curly, friable hair) and mental retardation [6]. Whether CFCS really represented a separate entity or just a variant of NS was a subject of debate for many years.

Another syndrome whose phenotypic overlap with NS has long been recognized is Costello syndrome (CS). This condition was first described in 1977 as a new syndrome with mental subnormality and nasal papillomata [7]. Patients with CS are short, have the same spectrum of congenital heart defects as NS and CFCS, and similar dysmorphism, although facial features are somewhat coarser than in the other 2 disorders (fig. 1c). Similarly to CFCS, most patients with CS are in the mentally handicapped range. They have soft and loose skin with deep palmar and plantar creases and sparse curly hair [8]. Most remarkable is a high risk of malignancy in CS, with embryonic rhabdomyosarcoma, neuroblastoma and bladder carcinoma being the most frequently reported tumors [9].

LEOPARD syndrome (LS) is an acronym for multiple lentigines, electrocardiographic conduction abnormalities, ocular hypertelorism, pulmonic stenosis, abnormal genitalia, retardation of growth and sensorineural deafness [10]. It is almost indistinguishable from NS in young children, with a similar pattern of heart defects and facial anomalies. The development of multiple pigmented skin lesions mostly starting in school age and the high prevalence of hearing deficits are typical of LS.

Finally, a fraction of patients with neurofibromatosis type 1 (NF1) also have obvious NS-like facial features with or without heart defects and short stature, prompting the classification as neurofibromatosis-Noonan syndrome for this apparently combined phenotype [11]. Notably, several features of NS also occur quite frequently in a milder expression in patients with NF1 not recognized as having neurofibromatosis-Noonan syndrome, such as a body height below the expected range, learning deficits, thorax deformity, facial anomalies, etc. [12]. The specific clinical characteristics of NF1 are skin pigmentation anomalies (multiple café-au-lait spots, lentigo-like freckling mainly of the axillary and inguinal regions) and the development of multiple tumors (particularly but not exclusively neurofibromas) that mostly affect the skin, but may also occur in other localizations. Recently, a NF1like syndrome characterized by multiple café-au-lait spots but absence of neurofibromas was described. Affected individuals may also show NS-like facial and other signs [13].

The recent insights into the common pathogenetic basis of these disorders have led to the suggestion to subsume them all under the term neuro-cardio-facial-cutaneous syndromes [14].

\section{RAS-MAPK Signaling Pathway}

RAS and the mitogen-activated protein kinases (MAPKs) RAF, MEK and ERK are involved in a ubiquitous signaling pathway that relays signals from receptor tyrosine kinases, such as growth factor receptors, to the 
nucleus (fig. 2). RAS proteins, encoded by the genes HRAS, KRAS and NRAS, act as molecular switches by cycling between active GTP-bound and inactive GDPbound states. Activated growth factor receptors recruit adaptor proteins that activate guanosine nucleotide exchange factors (GEFs), such as SOS1, to displace guanine nucleotides from RAS and permit passive binding to GTP. In the GTP-bound state, RAS undergoes a conformational change enabling the protein to bind and activate effector proteins such as RAF. This interaction is terminated by the release of a phosphate group from RAS.GTP to produce RAS $\cdot G D P$, restoring the inactive conformation. GTP hydrolysis is achieved by the intrinsic GTPase activity of RAS, which is augmented by GTPaseactivating proteins (GAPs), such as neurofibromin [15]. Activated RAS is also known to stimulate other effector pathways than RAF-MEK-ERK, such as PI3 kinase and RAL-GDS. Likewise, there is not only a single kind of signal input that is transmitted through RAS. Instead, RAS has to be regarded as one of the central nodes in the complex intracellular signaling network [14].

Abnormal RAS-MAPK signaling has long been known to be involved in tumorigenesis [16]. Constitutively activating somatic mutations of the RAS genes and $B R A F$ belong to the most common genetic events found in a broad spectrum of tumors, and, for example, account for about $60 \%$ of pancreatic and $35 \%$ of colon cancers (KRAS) as well as $40 \%$ of melanomas (BRAF) [16]. Mutations of the gene NF1, which encodes neurofibromin, a GAP for RAS, are known to underlie NF1. The tumor disposition in this disease further points to the oncogenic potency of deregulated RAS signaling. It is of note that JMML also belongs to the tumor spectrum associated with NF1 [4].

\section{Genetics of NS and Related Disorders}

From a formal genetic point of view, NS as well as the other disorders discussed here are inherited as an autosomal dominant trait with (almost) complete penetrance. However, more than half of the patients with NS and virtually all individuals affected with CFCS and CS represent sporadic cases due to de novo mutations. It has been demonstrated that de novo mutations predominantly occur on the paternally inherited allele and show a paternal age effect, indicating that these mutations may accumulate in sperm during lifetime [17]. In contrast, familial cases of NS are more often transmitted by the mother. This may reflect reduced reproductive fitness of males affected by NS. An autosomal recessive form of NS has

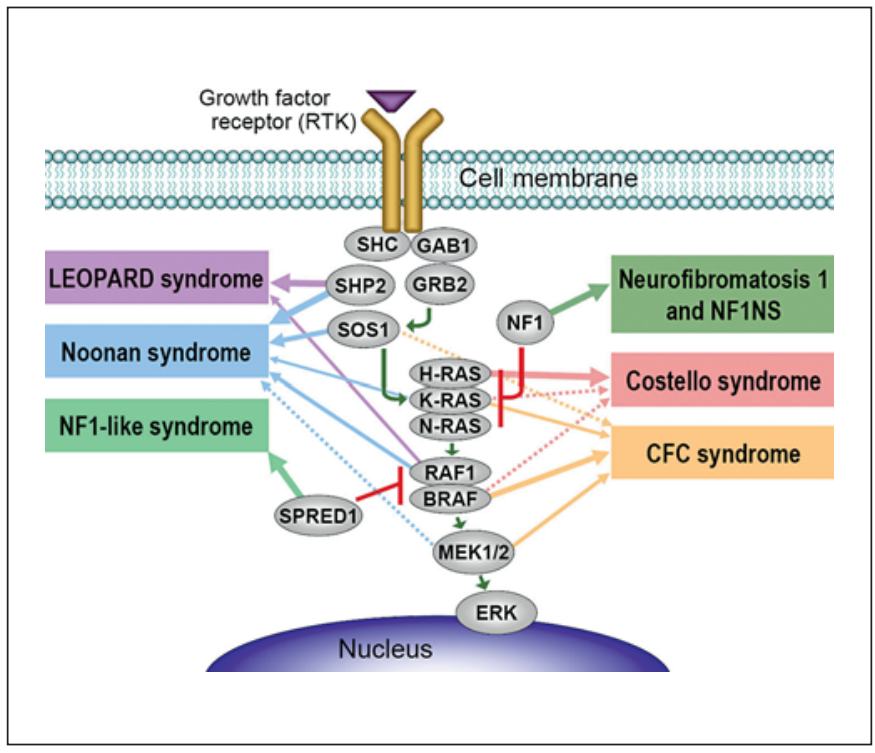

Fig. 2. RAS-MAPK signaling and disorders of the neuro-cardiofacial-cutaneous spectrum. Arrows point to the clinical entities related to the respective genes. Arrow width symbolizes the proportion of patients each gene accounts for. Dotted arrows indicate relationships reported only in single cases.

been discussed, but a clear proof of its existence is still lacking [18].

PTPN11, the gene encoding SHP2, was discovered as the first gene for NS in 2001 [19]. SHP2 is as a non-receptor protein-tyrosine phosphatase involved in the regulation of the phosphotyrosine content of specific intracellular proteins. Among its diverse functions, SHP2 acts as a positive regulator of RAS-MAPK signaling through mechanisms that are incompletely understood. At that time, however, the significance of the deregulation of RAS-MAPK signaling was not immediately recognized. Nevertheless, the association of NS with JMML prompted studies on somatic mutations in non-syndromic JMML, and PTPN11 was found to be mutated in up to one third of cases in this specific type of leukemia [20].

Another major breakthrough was achieved in 2005, when independent research groups almost simultaneously reported on the association of mutations in $R A S$ genes with disorders of the NS spectrum: mutations of HRAS were shown to be present in the vast majority ( $>90 \%)$ of patients with CS [21], and KRAS mutations were found in some cases of NS and CFCS [22]. At the same time, 2 other papers appeared, showing that altered signaling downstream of RAS due to mutations in BRAF, MEK1 
and MEK2 causes CFCS $[23,24]$. Together these findings definitely shifted the RAS-MAPK signaling pathway into the focus of subsequent research. Not surprisingly, other genes encoding molecules acting in this pathway were discovered to be involved in NS and related disorders. SOS1, whose gene product acts as a GEF for RAS, was found to be the second most commonly mutated gene in NS $[25,26]$, and RAF1 mutations were shown to be responsible for NS and LS [27, 28]. The most recent but likely not the last in this series of discoveries was the finding that mutations of SPRED1, encoding a negative regulator of RAS-MAPK signaling, cause an NF1-like disorder characterized by café-au-lait spots and variable NS-like features [13].

Those initial papers were drawing a picture of very clear genotype-phenotype correlations, with NS being associated with mutations in PTPN11, SOS1 and RAF1, while BRAF, MEK1 and MEK2 mutations cause CFCS. KRAS mutations appear to have a broader phenotypic spectrum, including both NS and CFCS. LS is consistently associated with specific PTPN11 mutations, but may also be caused by RAF1 mutations (fig. 2). Although these rules are useful and still valid for the vast majority of patients, there have been some more recent papers challenging a too-strict concept of genotype-phenotype correlations. NS patients with $M E K 1$ mutations were reported [29], as well as CFCS due to mutated SOS1 [30, 31]. Within the category of NS, there are also some specific phenotypic characteristics related to the underlying mutated gene. While short stature (below the 3rd centile for height) and learning difficulties appear to be somewhat less frequent in individuals with SOS1 mutations, they more often display ectodermal abnormalities that may be similar to those usually seen in CFCS $[26,32]$. RAF1 mutations are very strongly associated with hypertrophic cardiomyopathy $[27,28]$.

\section{Molecular Pathogenesis}

It is generally accepted that dysregulation of RASMAPK signaling (either increased or otherwise abnormal signal traffic) is the common pathophysiologic basis of all disorders of the neuro-cardio-facial-cutaneous spectrum [14]. This notion is supported by the fact that all mutations identified to date in agonists of the pathway (SHP2, SOS1, RAS, BRAF, RAF1, MEK1, MEK2) are missense changes and a few deletions of single amino acids clustering in certain functional domains of the respective proteins. In contrast, mutations in the negative regulators of the pathway, namely NF1 and SPRED1, include also nonsense, frame shift and splice site mutations predicting loss of function of the affected allele. The gain-of-function effects have been experimentally shown for selected SHP2, SOS1, KRAS, HRAS, BRAF, MEK1 and MEK2 mutants using eukaryotic cells over-expressing the mutant proteins and parameters, such as ERK phosphorylation as readout. Additional experiments have provided evidence that mutant proteins may be fixed in an active conformation or have increased catalytic activity [19, 21-23, 25-28]. However, the direct gainof-function effects could not be demonstrated for all mutants. In particular, PTPN11 mutations associated with LS showed decreased instead of increased catalytic function in those assays, and it was even proposed that these specific mutations may act in a dominant negative manner $[33,34]$. Mutants exhibiting decreased catalytic activity have also been detected in the BRAF and RAF1 genes $[24,27]$. This apparent paradox has not been completely resolved. Recent work, however, has elegantly shown in a Drosophila model that the biological consequences of LS mutations are anyhow similar to those of clearly activating mutations, and that they are related to the residual phosphatase activity of the mutant proteins [35]. These findings suggest that mechanisms other than the direct activation of the canonical pathway might be involved.

The question of whether and to what extent perturbed signaling, through other known RAS effector pathways or alteration of other SHP2 substrates, might contribute to the pathogenesis remains largely unsolved. Clearly, the identity of the genes found to be involved, to date, indicates that abnormal signal traffic through the RAF-MEKERK cascade is the most important pathophysiologic mechanism. A recent study has corroborated the concept that heart defect development in NS is indeed mediated through overactive ERK1, by showing that abnormal endocardial cushion formation can be induced by expressing $\mathrm{SHP} 2{ }^{\mathrm{Q} 79 \mathrm{R}}$ and rescued by ERK1 deletion in a mouse model [36]. However, this does not preclude disturbances in other RAS-dependent cascades being - at least in part - responsible for phenotypic differences between the specific entities. The precise pathophysiologic events leading to the specific organ manifestations of the NS group of disorders remain to be elucidated.

Regarding the pathophysiology of short stature in NS, it is of note that SHP2 has also been proposed to be involved in growth hormone signaling through the dephosphorylation of JAK2, leading to its inactivation and, consequently, decreased signaling through STAT5B [37]. 
This would be in line with findings indicating that NS patients carrying PTPN11 mutations may exhibit relative growth hormone resistance [38]. On the other hand, the presence of the same degree of short stature in patients with RAS, RAF and MEK mutations suggests that the main effect on growth is mediated by abnormal signaling through the RAS-MAPK pathway itself.

The linkage of this group of developmental disorders to an oncogenic pathway is particularly intriguing. Both somatic mutations in cancer as well as mutations occurring in the germline in NS and related disorders are presumed to lead to a constitutional activation of signal traffic. Nevertheless, the tumor risk in most of these conditions is quite low. Notably, for SHP2, KRAS and BRAF, it is evident that oncogenic and germline mutations may cluster in the same functional domains, but they do not really overlap [39-41]. Some studies have suggested that those mutations occurring in the germline are hypomorphs compared to the oncogenic mutations, suggesting a quantitative difference in activation potential as the main factor determining the clinical consequences $[22,34]$. It has been speculated that 'oncogenic' mutations occurring in the germline might lead to embryonic lethality [42]. Support for this view comes from the very recent observation of oncogenic PTPN11 mutations in spontaneous abortions [43]. On the other hand, those mutations that are viable in the germline have been suggested to confer a low degree of activation or transforming potential that is unlikely to promote tumorigenesis [42]. While the concept of a different degree of activation between oncogenic and germline mutations is widely accepted, in principle, it is possibly an oversimplification, and qualitative differences or cellspecific or other effects may also have to be considered.

Furthermore, some exceptions do exist: a few specific germline mutations, such as PTPN11 ${ }^{\mathrm{T} 73 \mathrm{I}}$ and $K R A S^{\mathrm{T} 58 \mathrm{I}}$, have been found to be particularly associated with NS plus JMML $[22,39,44]$. The most important exception is CS, where the same HRAS mutations known as oncogenic are present in the germline. This fact obviously provides an explanation for the high tumor risk that is specific for CS. On the other hand, it suggests a less critical role of HRAS during embryonic development. A strong propensity to tumor development is also typical for NF1. It has been suggested that in NF1 the defect of one NF1 allele in the germline may cause some mild deregulation of RAS, similar to or even milder than the effect of mildly activating mutations in the pathway's agonists in NS. This mild but generalized alteration of signaling is prob- ably responsible for the variable NS-like features, including cognitive and growth deficits in NF1. In contrast, tumor development is dependent on a somatic second hit affecting the other allele, eventually resulting in strong over-activation of RAS in the affected cell and promoting clonal proliferation [11].

\section{Perspectives}

Currently, the genetic basis remains undiscovered in about $20-30 \%$ of cases with NS, $15-20 \%$ of CFCS and a small fraction of patients with a clinical diagnosis of CS. It is likely that mutations in other molecules related to the RAS-MAPK pathway are responsible for these cases. Studies aimed at the identification of further genes for NS and related disorders are ongoing in several research groups. Moreover, animal models are being established in order to increase the understanding of molecular pathogenesis and mechanisms leading to abnormal development. One of the most exciting issues related to the developmental disorders of the RAS-MAPK pathway is the idea that a causal treatment of at least some of their manifestations might become possible in future. The hopes and expectations are related to the fact that substances having the potential to inhibit RAS-MAPK signaling are already known and available. Inhibitors of RAF and MEK, such as sorafenib and PD-325901, respectively, as well as farnesyltransferase inhibitors, such as lonafarnib, have been developed because of their proposed effect as anticancer drugs. A few of them are already in clinical use. However, significant side effects make them unsuitable as a long-term (maybe life-long) medication. Another substance class with a known inhibitory effect on RAS activation is the HMG-CoA reductase inhibitors (statins), which are well-known as cholesterol-lowering drugs. Through their effects in cholesterol anabolism, they also interfere with farnesylation and geranylgeranylation of RAS, which is important for its anchorage to the plasma membrane, the site of their activation. Thus, decreased post-translational modification of RAS may lower the fraction of functionally available RAS. Lovastatin treatment has been shown to restore the learning deficits in an NF1 mouse model [45]. Treatment trials in NF1 patients are underway. One pilot study has recently been published with only marginal improvement in the treatment group [46], but other studies are ongoing. While statins might work in conditions with over-activation upstream from RAS, theoretically, they may be inefficient in CFCS due to mutations of BRAF or 
MEK. Suitable outcome measures, dosages, and start and duration of treatment still need to be defined. Some experts in the field argue that studies in animal models have to be completed first, before clinical trials are set up. Despite all unsolved problems and ongoing discussions, NS and related conditions may become the first genetic disorders of development that are responsive to medical treatment.

\section{Disclosure Statement}

The author declares that there are no conflicts of interest.

\section{References}

1 Noonan JA: Hypertelorism with Turner phenotype: a new syndrome with associated congenital heart disease. Am J Dis Child $1968 ; 116: 373-380$

2 Allanson JE: Noonan syndrome. Am J Med Genet C Semin Med Genet 2007;145C:274279.

3 van der Burgt I: Noonan syndrome. Orphanet J Rare Dis 2007;2:4.

4 Kratz CP, Niemeyer CM: Juvenile myelomonocytic leukemia. Hematology 2005; 10(suppl 1):100-103.

5 Reynolds JF, Neri G, Herrmann JP, et al: New multiple congenital anomalies/mental retardation syndrome with cardio-facio-cutaneous involvement - the CFC syndrome. Am J Med Genet 1986;25:413-427.

6 Roberts A, Allanson J, Jadico SK, et al: The cardiofaciocutaneous syndrome. J Med Genet 2006;43:833-842.

7 Costello JM: A new syndrome: mental subnormality and nasal papillomata. Aust Paediatr J 1977;13:114-118.

8 Hennekam RC: Costello syndrome: an overview. Am J Med Genet C Semin Med Genet 2003;117:42-48.

9 Gripp KW: Tumor predisposition in Costello syndrome. Am J Med Genet C Semin Med Genet 2005;137:72-77.

10 Sarkozy A, Digilio MC, Dallapiccola B: LEOPARD syndrome. Orphanet J Rare Dis 2008;3:13

11 Huffmeier U, Zenker M, Hoyer J, et al: A variable combination of features of Noonan syndrome and neurofibromatosis type I are caused by mutations in the NF1 gene. Am J Med Genet A 2006;140:2749-2756.

12 Colley A, Donnai D, Evans DG: Neurofibromatosis/Noonan phenotype: a variable feature of type 1 neurofibromatosis. Clin Genet 1996;49:59-64.

13 Brems H, Chmara M, Sahbatou M, et al Germline loss-of-function mutations in SPRED1 cause a neurofibromatosis 1-like phenotype. Nat Genet 2007;39:1120-1126.

14 Bentires-Alj M, Kontaridis MI, Neel BG: Stops along the RAS pathway in human genetic disease. Nat Med 2006;12:283-285.
15 Wittinghofer A: Signal transduction via Ras. Biol Chem 1998;379:933-937.

16 Bos JL: ras oncogenes in human cancer: a review. Cancer Res 1989;49:4682-4689.

17 Tartaglia M, Cordeddu V, Chang H, et al: $\mathrm{Pa}-$ ternal germline origin and sex-ratio distortion in transmission of PTPN11 mutations in Noonan syndrome. Am J Hum Genet 2004; 75:492-497.

18 van Der Burgt I, Brunner H: Genetic heterogeneity in Noonan syndrome: evidence for an autosomal recessive form. Am J Med Genet 2000;94:46-51.

19 Tartaglia M, Mehler EL, Goldberg R, et al: Mutations in PTPN11, encoding the protein tyrosine phosphatase SHP-2, cause Noonan syndrome. Nat Genet 2001;29:465-468.

20 Tartaglia M, Niemeyer CM, Fragale A, et al: Somatic mutations in PTPN11 in juvenile myelomonocytic leukemia, myelodysplastic syndromes and acute myeloid leukemia. Nat Genet 2003;34:148-150.

21 Aoki Y, Niihori T, Kawame H, et al: Germline mutations in HRAS proto-oncogene cause Costello syndrome. Nat Genet 2005; 37:1038-1040.

22 Schubbert S, Zenker M, Rowe SL, et al: Germline KRAS mutations cause Noonan syndrome. Nat Genet 2006;38:331-336.

23 Niihori T, Aoki Y, Narumi Y, et al: Germline $K R A S$ and $B R A F$ mutations in cardio-faciocutaneous syndrome. Nat Genet 2006;38: 294-296.

24 Rodriguez-Viciana P, Tetsu O, Tidyman WE, et al: Germline mutations in genes within the MAPK pathway cause cardio-facio-cutaneous syndrome. Science 2006;311:1287-1290.

25 Roberts AE, Araki T, Swanson KD, et al: Germline gain-of-function mutations in SOS1 cause Noonan syndrome. Nat Genet 2007;39:70-74

26 Tartaglia M, Pennacchio LA, Zhao C, et al: Gain-of-function SOS1 mutations cause a distinctive form of Noonan syndrome. Nat Genet 2007;39:75-79.

27 Pandit B, Sarkozy A, Pennacchio LA, et al: Gain-of-function RAF1 mutations cause Noonan and LEOPARD syndromes with hypertrophic cardiomyopathy. Nat Genet 2007; 39:1007-1012.
28 Razzaque MA, Nishizawa T, Komoike Y, et al: Germline gain-of-function mutations in RAF1 cause Noonan syndrome. Nat Genet 2007;39:1013-1017.

29 Nava C, Hanna N, Michot C, et al: Cardiofacio-cutaneous and Noonan syndromes due to mutations in the RAS/MAPK signalling pathway: genotype-phenotype relationships and overlap with Costello syndrome. J Med Genet 2007;44:763-771.

30 Narumi Y, Aoki Y, Niihori T, et al: Clinical manifestations in patients with SOS1 mutations range from Noonan syndrome to CFC syndrome. J Hum Genet 2008;53:834-841.

31 Nystrom AM, Ekvall S, Berglund E, et al: Noonan and cardio-facio-cutaneous syndromes: two clinically and genetically overlapping disorders. J Med Genet 2008;45: 500-506.

32 Zenker M, Horn D, Wieczorek D, et al: SOS1 is the second most common Noonan gene but plays no major role in cardio-facio-cutaneous syndrome. J Med Genet 2007;44:651656.

33 Kontaridis MI, Swanson KD, David FS, et al: PTPN11 (Shp2) mutations in LEOPARD syndrome have dominant negative, not activating, effects. J Biol Chem 2006;281:67856792.

34 Tartaglia M, Martinelli S, Stella L, et al: Diversity and functional consequences of germline and somatic PTPN11 mutations in human disease. Am J Hum Genet 2006;78: 279-290.

35 Oishi K, Zhang H, Gault WJ, et al: Phosphatase-defective LEOPARD syndrome mutations in PTPN11 have gain-of-function effects during Drosophila development. Hum Mol Genet 2008;18:193-201.

36 Krenz M, Gulick J, Osinska HE, et al: Role of ERK1/2 signaling in congenital valve malformations in Noonan syndrome. Proc Natl Acad Sci USA 2008;105:18930-18935.

37 Kim SO, Jiang J, Yi W, et al: Involvement of the Src homology 2-containing tyrosine phosphatase SHP-2 in growth hormone signaling. J Biol Chem 1998;273:2344-2354. 
38 Binder G, Neuer K, Ranke MB, et al: PTPN11 mutations are associated with mild growth hormone resistance in individuals with Noonan syndrome. J Clin Endocrinol Metab 2005;90:5377-5381.

39 Aoki Y, Niihori T, Narumi Y, et al: The RAS/ MAPK syndromes: novel roles of the RAS pathway in human genetic disorders. Hum Mutat 2008;29:992-1006.

40 Schulz AL, Albrecht B, Arici C, et al: Mutation and phenotypic spectrum in patients with cardio-facio-cutaneous and Costello syndrome. Clin Genet 2008;73:62-70.

41 Zenker M, Lehmann K, Schulz AL, et al: Expansion of the genotypic and phenotypic spectrum in patients with KRAS germline mutations. J Med Genet 2007;44:131-135.
42 Kratz CP, Niemeyer CM, Zenker M: An unexpected new role of mutant Ras: perturbation of human embryonic development. J Mol Med 2007;85:227-235.

43 Lee K, Williams B, Roza K, et al: PTPN11 analysis for the prenatal diagnosis of Noonan syndrome in fetuses with abnormal ultrasound findings. Clin Genet 2009;75:190194.

44 Kratz CP, Niemeyer CM, Castleberry RP, et al: The mutational spectrum of PTPN11 in juvenile myelomonocytic leukemia and Noonan Syndrome/myeloproliferative disease. Blood 2005;106:2183-2185.

45 Li W, Cui Y, Kushner SA, et al: The HMGCoA reductase inhibitor lovastatin reverses the learning and attention deficits in a mouse model of neurofibromatosis type 1. Curr Biol 2005;15:1961-1967.
46 Krab LC, de Goede-Bolder A, Aarsen FK, et al: Effect of simvastatin on cognitive functioning in children with neurofibromatosis type 1: a randomized controlled trial. JAMA 2008;300:287-294.

\section{Note Added in Proof}

After acceptance of this paper for publication, a specific mutation of SHOC2, which encodes a modulator of RAS-MAPK signaling, was reported to cause a clinically relatively homogeneous variant of NS named Noonan-like syndrome with loose anagen hair [Cordeddu et al., Nat Genet 2009;41(9):1022-1026]. 